Vorschriften dieser Methode beigefügt werden: »Süssweine muss man vor dem Fällen mit Aether-Alkoholgemisch vollständig vergähren lassen.«

Der von Petrowitsch angezogene Satz aus dem Buche von Dr. Oscar Dietzsch ( Die wichtigsten Nahrungsmittel und ihre Fälschungen, « IV. Auflage, pag. 115): »Hat sich gar kein Weinstein nach 24 Stunden abgeschieden, so ist der Wein unzweifelhaft als Kunstwein zu betrachten, « ist zwar für sehr stark gegypste Weine zutreffend, indem bei übermässig gegypsten Weinen aller Weinstein als weinsteinsaurer Kalk ausgefällt sein kann. Diese Weine werden als übermässig gegypst beanstandet, können aber nicht als Kunstwein bezeichnet werden.

Genf, Laboratoire du Bureau de Salubrité, März 1892.

\title{
Versuch eines Nachweises fremder Fette in der Butter.
} Von

\section{Dr. J. Erdélyi.}

Der Nachweis fremder, in gewinnsüchtiger Absicht beigemengter Fette zur Butter ist zweifelsohne eine für den Nahrungsmittelchemiker eben so wichtige als - leider nur zu häufig — schwer in eindeutigem Sinne zu lösende Aufgabe. All' die Methoden qualitativer und quantitativer Natur, durch deren Combination es zumeist gelingen wird, Mischungen von pflanzlichen und thierischen Fetten oder Mineralölen als solche zu erkennen, lassen uns hier oft im Stich. Auch die Reichert-Meissl'sche Zahl, welche in der ersten Zeit ihrer Anwendung einen verlässlichen Anhaltspunkt in dieser Beziehung abzugeben versprach, scheint den gehegten Erwartungen nicht entsprochen zu haben, denn von dem anfänglich stipulirten Grenzwerth von $26 c c^{1 / 10^{-N o r m a l-}}$ lauge für $5 g$ Butterfett ${ }^{1}$ ) ist man im Laufe der Jahre immer mehr ab- und zurückgewichen, so Meissl selbst auf $24 c c$, dann Andere auf 22, 20, $18 c c$, ja unter diesem Werthe wollen Analytiker Butterproben in der Hand gehabt haben. ${ }^{2}$ )

Unter solchen Umständen musste eine bedauerliche Unsicherheit in der Beurtheilung und andererseits geradezu eine Vorschubleistung $\gg$ ratio-

1) Untersuchungen über die VerfäIschungen des käuflichen Butterschmalzes von Dr. E. Meiss1, Dingler's polyt. Journal 223, 229 (1879). Vergl. auch diese Zeitschrift 18, $68 \mathrm{ff}$.

2) Chemiker-Zeitung Repert. 1891, S. 195. 
neller « Butterverfälschung platzgreifen, Missstände, zu deren Behebung, wie die periodische Fachlitteratur der letzten Jahre beweist, eine stattliche Reihe von Versuchen angestellt worden ist, doch wie es scheint ohne durchgreifenden Erfolg. Wenn ich nun trotzdem Veranlassung nahm, zur Lösung dieser Frage auch meinerseits beizutragen, so geschah es in der Hoffnung, dass es durch zweckentsprechende Modification einer bereits angewandten und bewährten Methode gelingen könnte, das angestrebte Ziel zu erreichen; es ist dies die zur Identitäts- und Reinheitsbestimmung des Cacaofettes benutzte Björklund'sche Probe, welche bekanntlich darauf basirt, dass reines Cacaofett in ätherischer Lösung von bestimmter Concentration, der Temperatur von $0^{0}$ ausgesetzt, sich eine gewisse Zeit hindurch unverändert erhält, während durch fremde Zusätze verfälschtes sich mehr oder minder rasch trübt.

Versuche, die ich auf Grundiage dieses Principes vergangenes Jahr in Göttingen mit den verschiedensten Lösungsmitteln und deren Gemischen angestellt habe, schlugen zumeist fehl; erst als das in der mikroskopischen Technik als Lösungsmittel angewandte Xylol herangezogen wurde, gestaltete sich die Sache aussichtsvoller; obschon es in einer Anzahl von Fällen gelang, recht auffällige Unterschiede im Verhalten von Butter und Butter-Margaringemischen zu beobachten, so befriedigten mich die Resultate zu wenig, als dass eine praktische Brauchbarkeit der Methode angenommen werden konnte.

In Folge Stellungswechseis und Inanspruchnahme konnten die diesbezüglichen Versuche erst nach Jahresfrist wieder aufgenommen werden; ich wähnte mich durch Anwendung der höheren Benzolhomologen auf gutem Wege -, es handelte sich nur darum, durch Ausfindigmachen des geeigneten Lösungsmittels und der Coucentration, der Methode so weit als möglich - eine sichere Grundlage zu geben. Nach mehrfachem Probiren glaube ich im $\mathrm{Cumol}^{1}$ ), und zwar in der Concentration von $2 c c$ Fett in $6 c c \mathrm{Cumol}^{2}$ ) das Gewünschte gefunden zu haben.

Auf Grund meiner Versuche glaube ich zur Annahme berechtigt zu sein, dass eine auf diese Weise bereitete Lösung von reinem Butterfett, einer Temperatur von $0^{\circ}$ ausgesetzt, sich mindestens eine Stunde hindurch unverändert klar hält. Wie aus unten stehenden An-

1) Von E. Merck, Darmstadt. Siedepunkt 165 bei $758 \mathrm{~mm}$ Bar.

2) Anfangs arbeitete ich mit Grammen, später verwendete ich in Interesse des rascheren Arbeitens Volumina. 
gaben ersichtlich, wird diese Zeitdauer zumeist ganz erheblich übertroffen. In allen beobachteten Fällen nun war der Zeitunterschied der eintretenden Trübung der Butterlösung einerseits und der Lösung der Fettgemische andererseits ein so beträchtlicher, der Zustand der betreffenden Lösungen ein so augenfällig verschiedener, dass eine sichere Diagnose keinem Zweifel unterliegen konnte.

Es sei mir gestattet, an der Hand einiger Beobachtungsresultate das oben geschilderte Verhalten näher zu beleuchten, vorerst jedoch über Art und Weise der Versuchsanstellung einige Worte zu sagen. Das klar filtrirte Fett, respective Fettgemisch, wurde 24-48 Stunden hindurch an einem kühlen Ort (Eisschrank) sich selbst überlassen, dann wurden je $2 c c$ in peinlichst gereinigte, trockene Eprouvetten möglichst gleichen Calibers gebracht (ich verwandte solche mit $2 \mathrm{~cm}$ innerer Lichte), $6 c c$ Cumol zugesetzt und bei Zimmertemperatur wieder 24 Stunden stehen gelassen. Nun wurden die so vorbereiteten Eprouvetten - nebst Thermometer - zwischen klein gestossenes Eis gebracht; eine Stunde hingestellt und dann in gewissen Zeitintervallen zur Besichtigung auf einen Augenblick herausgehoben. Der Nachtheil der langen Dauer, welcher. der. Methode vorlänfig anhaftet, wird sich vielleicht beheben lassen, weitere Versuche in dieser Richtung behalte ich mir vor. Nun einige aus meinen Notizen excerpirte Daten:

\section{Reichert- \\ Me is sl'sche}

Zahl

Süssrahmbutter von W. . . . . . 28,8 Cumollösung nach $11 / 2$ St.ganz klar. Gemisch dieser Butter und Margarine . 22,2, \# "1 Stde. schwach, nach weiteren 10 Min. ganz trüb.

Sanerrahmbutter von W. . . . . 27,5

und Margarine. . 21,5

\#Schweinefett . . 22,4

Butterfett aus Rahm:

süss abgerahmt. . . . . . . . 29,8

, $\quad$ und Margarine . . 22,4

$" \quad \quad \quad$ Schweinefett . . 26,3

sauer abgerahmt . . . . . . 29,8

und Margarine. . . 22,2

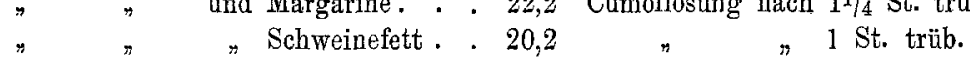

Cumollösung nach 1 Stunđe trüb.

"2 Stunden klar.

"11/4 St. trüb.

$\rightarrow$

$n$

" $1^{1 / 2}$ St. klar.

"11/4 n beginnende Trübung, nach" weiteren 10 Min. ganz trüb.

Cumollösung schon beim Herausheben, also innerhalb 1 St. trüb. Camollösung während der beobachteten 2 Stunden klar.

Cumollösung nach $1^{1 / 4}$ St. trüb. 
Die angeführten, sowie alle übrigen Beobachtungen wurden stets von Unbetheiligten controlirt, zum Theil habe ich Proben signiren lassen und stets mit Sicherheit das reine Butterfett von den Mischungen unterscheiden können.

Gewiss unterliegt es keinem Zweifel, dass die Ergebnisse einer immerhin kleinen Anzahl von Beobachtungen durchaus keine Gewähr für die Brauchbarkeit der Methode abgeben; wenn ich diese Versuche den Fachgenossen dennoch zur Kenntniss bringe, so ist es eben die Ueberzeugung, dass Mittel und Kräfte des Einzelnen zur raschen und sicheren Lösung dieser Frage unzureichend sind, diese vielmehr nur durch eine möglichst vielseitige Versuchsanstellung mit authentischem Materiale verschiedener Provenienz zu erhoffen ist; diese einfache, wenig Arbeit und Material erfordernde Methode scheint nach dem Vorgebrachten eines Versuches werth $\mathrm{zu}$ sein.

Ma inz, Chemisches Untersuchungsamt für die Provinz Rheinhessen.

Ueber einen Apparat zum gleichförmigen Vermischen grösserer Mengen pulverförmiger Körper.

\section{Von \\ C. Mann.}

Die Herstellung eines gleichförmigen Gemenges verschiedenartiger und auf möglichst gleiches Korn gebrachter Substanzen - in grösseren Mengen - zu ein und mehreren Kilogrammen ist nicht so gar ausserordentlich einfach und leicht.

Bei öfterem Anhäufen $»$ auf den Kegel « und nachherigem Vermischen mit dem Löffel - wie dies gewöhnlich zu geschehen pflegt gibt es kein Kennzeichen, um sofort beurtheilen zu können, wie oft diese Procedur vorgenommen werden soll und wann eben eine herauszunehmende verjüngte Probe die Qualität der ganzen Masse darstellt.

Beim Zerreiben und Vermengen von nur wenigen Grammen in der Achat- oder Porzellanreibschale wird wohl kein $Z_{w}$ eifel in dieser Art obwalten, aber ganz besonders wird dieser Umstand von Bedeutung, wenn es sich z. B. darum handelt, sehr kleine Mengen in sehr grosse Massen einer anderen Substanz gleichförmig einzuverleiben, oder wenn die Aufgabe gestellt wird, von mehreren hundert oder tausend im Jahre 